\title{
PHRAGMÉN-LINDELÖF THEOREM FOR THE MINIMAL SURFACE EQUATION
}

\author{
JENN-FANG HWANG
}

(Communicated by Walter Littman)

\begin{abstract}
It is proved that if $u$ satisfies the minimal surface equation in an unbounded domain $\Omega$ which is properly contained in a half plane, then the growth property of $u$ depends on $\Omega$ and the boundary value of $u$ only.
\end{abstract}

1. Introduction. The purpose of this paper is to establish a PhragménLindelöf Theorem for the minimal surface equation in $\mathbf{R}^{2}$. We prove that if $u$ satisfies the minimal surface equation in an unbounded domain $\Omega$, which is properly contained in a half plane, then the growth property of $u$ depends on $\Omega$ and $\left.u\right|_{\partial \Omega}$ only, without requiring any other restriction for $u$. In this respect, the PhragménLindelöf Theorem for the minimal surface equation is better than that of the linear equations. We remark that if $u$ satisfies the Laplace equation with vanishing boundary value in a sector domain $\Omega_{\alpha}$ with angle $0<\alpha<\pi$, then we cannot conclude that $u \equiv 0$. If we want to establish a maximum principle on $\Omega_{\alpha}$, we must impose some restriction on the growth of $u$ at infinity [12, Chapter 2, §9]. In fact, for the Laplace equation in an unbounded domain $\Omega$, the growth property of $u$ cannot be determined completely by $\left.u\right|_{\partial \Omega}$ alone. There are various types of restriction on the growth of $u$ at infinity in Phragmén-Lindelöf Theorems for general equations. For these results, the reader is referred to $[\mathbf{1}, \mathbf{2}, \mathbf{3}, \mathbf{5}, \mathbf{6}, \mathbf{7}, \mathbf{1 0}, \mathbf{1 2}, \mathbf{1 3}]$.

On the other hand, if $u$ satisfies the minimal surface equation with vanishing boundary value in $\Omega_{\alpha}$, then $u \equiv 0[8, \mathrm{p} .256]$. So it is natural to conjecture that if $u$ satisfies the minimal surface equation in $\Omega_{\alpha}$, then the growth property of $u$ depends on $\left.u\right|_{\partial \Omega}$ only.

We prove that if $\Omega \subset\{(x, y) \mid y>0,-f(y)<x<f(y)\}$ where $f \in C^{0}[0, \infty)$, $f \geq 0, f(t)$ increases as $t$ increases, then the previous conjecture is true (Main Theorem). Our estimates depend on the shape of $\Omega$, and the behavior of $\left.u\right|_{\partial \Omega}$ does not enter the picture explicitly.

We emphasize that in such a domain $\Omega$, the solutions for the minimal surface equation with vanishing boundary value may not be unique, but the Main Theorem is still true. (e.g. in $\Omega=\left\{(x, y) \mid-\sqrt{(\cosh y)^{2}-1}<x<\sqrt{(\cosh y)^{2}-1}, y>0\right\}$, we have two solutions with vanishing boundary value $u \equiv 0$ and $u=\sqrt{(\cosh y)^{2}-x^{2}}-$ 1). Since in a half plane, the bound of the solutions with vanishing boundary value does not even exist, the domain must be properly contained in a half plane.

Some examples and remarks can be found in $\S 3$.

Received by the editors October 3, 1986 and, in revised form, December 1, 1987. The result in this paper was announced in "Colloque Sino-Franscais sur les equations differentielles" held in Hsincher, Taiwan, R.O.C., on April 8, 1988.

1980 Mathematics Subject Classification (1985 Revision). Primary 35J60, 35J15, 35B40, 53A10.

This research was supported by the National Science Council, R.O.C. 
2. Main theorem. Throughout the whole article, $\Omega$ will be a connected domain (bounded or unbounded) in $\mathbf{R}^{2}$, and for any function $u \in C^{1}(\Omega), T u$ will denote the vector $D u / \sqrt{1+|D u|^{2}}$ where $D u$ is the gradient vector of $u$.

For latter arguments of comparison, a particular function will be utilized. Its definition and basic properties are stated in the following lemma whose proof follows by direct computation:

LEMMA 2.1. Let $v=(1 / c) \cdot\left(x^{2}-x_{0}^{2}\right) /\left(y-y_{0}\right)+a\left(y-y_{0}\right)+b$ in $\Omega=\left(-x_{0}, x_{0}\right) \times$ $\left(-\infty, y_{0}\right)$ where $c, a, x_{0}$ are positive constants and $b, y_{0}$ are constants. Then we have

(i) $\lim _{y \rightarrow y_{0}} v=+\infty$,

(ii) $\operatorname{div} T v \leq 0$ in $\Omega$ for $0<c \leq 4 a$,

(iii) $v(x, y) \geq v\left(x_{0}, y\right)=v\left(-x_{0}, y\right)$ for every $y<y_{0}$ and $x \in\left(-x_{0}, x_{0}\right)$.

Now, we have

THEOREM 2.2. Let $\Omega \subset\left(-x_{0}, x_{0}\right) \times\left(0, y_{0}\right)$ and let $u \in C^{0}(\bar{\Omega}) \cap C^{2}(\Omega)$. Suppose that

(i) $\operatorname{div} T u \geq 0$ in $\Omega$,

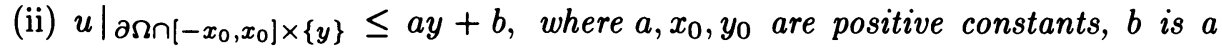
constant, $0 \leq y<y_{0}$.

Then if $y_{0}-x_{0} /(2 a)>0$, we have $u(x, y) \leq a y_{0}+b$ for every $(x, y) \in\left(-x_{0}, x_{0}\right) \times$ $\left(0, y_{0}-x_{0} /(2 a)\right) \cap \Omega$.

ProOF. Let $v=(1 /(4 a)) \cdot\left(x^{2}-x_{0}^{2}\right) /\left(y-y_{0}\right)+a y+b$. Since $\left.u\right|_{\partial \Omega} \leq\left. v\right|_{\partial \Omega}$ and $\operatorname{div} \mathrm{Tu} \geq 0 \geq \operatorname{div} \mathrm{Tv}$ in $\Omega$, we have $u \leq v$ in $\Omega$. Noting that $v(x, y) \leq a y_{0}+b$ for every $(x, y) \in\left(-x_{0}, x_{0}\right) \times\left(0, y_{0}-x_{0} /(2 a)\right) \cap \Omega$, one immediately completes the proof.

REMARK. Since

$$
\left.v\right|_{\left(-x_{0}, x_{0}\right) \times\left\{y_{0}\right\}}=+\infty,
$$

there are no restrictions for $\left.u\right|_{\partial \Omega \cap\left[-x_{0}, x_{0}\right] \times\left\{y_{0}\right\}}$. The Main Theorem follows from this idea:

MAIN THEOREM. Let $\Omega \subset \Omega_{1}=\{(x, y) \mid y>0,-f(y)<x<f(y)\}$ where $f, g \in C^{0}[0, \infty), f, g \geq 0, g(0)=0, f(t), g(t) / t$ increase as $t$ increases, and let $u \in C^{0}(\bar{\Omega}) \cap C^{2}(\Omega)$. Suppose that

(i) $\operatorname{div} T u \geq 0$ in $\Omega$,

(ii) $\left.u\right|_{\partial \Omega \cap[-f(y), f(y)] \times\{y\}} \leq g(y)$ where $y \in[0, \infty)$,

(iii) $0<\beta(y) \equiv f(y) /(2 g(y))<1$ for some $y_{1}>0$ and every $y>y_{1}$,

(iv) $\beta(y)$ decreases in $\left[y_{1}, \infty\right)$.

Then $u(x, y) \leq g(y /(1-\beta(y)))$ for every $(x, y) \in \Omega$ where $y>y_{1}$.

ProOF. Fixing $y_{2}>y_{1}>0$ and noting that for every $0 \leq y<y_{2}$, we have $\left.u\right|_{\partial \Omega \cap[-f(y), f(y)] \times\{y\}} \leq g(y) \leq\left(g\left(y_{2}\right) / y_{2}\right) y$. By Theorem 2.2 , we have $u(x, y) \leq g\left(y_{2}\right)$ where $0 \leq y \leq y_{2}-\beta\left(y_{2}\right) y_{2}$. Now for every $y_{3}>y_{1}$, we have $y_{3} /\left(1-\beta\left(y_{3}\right)\right)>y_{3}>y_{1}$ and $y_{3}=\left(1-\beta\left(y_{3}\right)\right) y_{3} /\left(1-\beta\left(y_{3}\right)\right) \leq y_{3} /\left(1-\beta\left(y_{3}\right)\right)-$ $\beta\left(y_{3} /\left(1-\beta\left(y_{3}\right)\right)\right) y_{3} /\left(1-\beta\left(y_{3}\right)\right)$, and we obtain $u\left(x, y_{3}\right)<g\left(y_{3} /\left(1-\beta\left(y_{3}\right)\right)\right)$.

REMARK. In the above theorem, $f$ controls the increasing rapidity of width of the defined domain $\Omega$ and $g$ controls the increasing rapidity of boundary value. It is natural that we require $g(y) / y$ to increase as $y$ increase. 
3. Examples and remarks. Now, we give some examples for the applications of the Main Theorem.

EXAMPLE 3.1. Let $\Omega=\{-y<x<y \mid y>0\}$ and let $u \in C^{0}(\bar{\Omega}) \cap C^{2}(\Omega)$. Suppose that $\operatorname{div} T u \geq 0$ in $\Omega$ and $u( \pm y, y) \leq y^{m}$ for every $y \geq 0$, where $m>1$ is a positive constant. Then for $y \geq 1$, we have $\beta(y)=\left(2 y^{m-1}\right)^{-1} \leq 1$, and $u(x, y) \leq\left(y /\left(1-\left(2 y^{m-1}\right)^{-1}\right)^{m}=y^{m}+(m / 2) y+O\left(y^{2-m}\right)\right.$ as $y \rightarrow \infty$.

REMARK 3.2. As [8, p. 256], with the help of the general maximum principle and a suitable solution of Dirichlet's problem [8, II, 7.2] the following maximum principle can be proved: Let $\Omega=\{-y<x<y \mid y>0\}$ (or a sector domain $\Omega_{\alpha}$ with angle $0<\alpha<\pi$ ), and let $u \in C^{0}(\bar{\Omega}) \cap C^{2}(\Omega)$ be a solution of the minimal surface equation in $\Omega$. Then

(i) if $u( \pm y, y) \leq a y+c$, we have $u(x, y) \leq a y+c$,

(ii) if $u( \pm y, y) \geq a y+c$, we have $u(x, y) \geq a y+c$,

where $a$ and $c$ are constants. Further,

(iii) if $u( \pm y, y) \leq g(y)$ where $g(y) \in C^{0}[0, \infty) \cap C^{2}(0, \infty)$ and $g^{\prime \prime}(y) \leq 0$ for every $y>0$, we have $u(x, y) \leq g(y)$,

(iv) if $u( \pm y, y) \geq h(y)$ where $h(y) \in C^{0}[0, \infty) \cap C^{2}(0, \infty)$ and $h^{\prime \prime}(y) \geq 0$ for every $y>0$, we have $u(x, y) \geq h(y)$.

(In (iii), we have $u( \pm y, y) \leq g\left(y_{0}\right)+g^{\prime}\left(y_{0}\right)\left(y-y_{0}\right)$ for every fixing $y_{0}>0$, by (ii) $u(x, y) \leq g\left(y_{0}\right)+g^{\prime}\left(y_{0}\right)\left(y-y_{0}\right)$ and $u\left(x, y_{0}\right) \leq g\left(y_{0}\right)$.)

In Example 3.1, if $u( \pm y, y) \leq y^{m}$ where $0<m \leq 1$ is a constant, by (iii) we have $u(x, y) \leq y^{m}$, but the Main Theorem will not give the optimal result.

In Example 3.1, if $u( \pm y, y) \leq y^{m}$ where $1<m$ is a constant, by (iv) $u(x, y)$ may be greater than $y^{m}$, it seems that $u(x, y)=y^{m}+(m / 2) y+O\left(y^{2-m}\right)$ is a good estimate.

Now, we give two examples to explain how to estimate the solutions with vanishing boundary value.

EXAMPLE 3.3. Let $\Omega=\{-\sinh y<x<\sinh y \mid y>0\}$ and let $u \in C^{0}(\bar{\Omega}) \cap$ $C^{2}(\Omega)$. Suppose that $\operatorname{div} T u \geq 0$ in $\Omega$ and $\left.u\right|_{\partial \Omega} \leq 0$. Consider $g(y)=c \sinh y$, where $c>\frac{1}{2}$ is a constant to be specified. Then $\beta(y)=1 / 2 c<1$ and $u(x, y) \leq$ $c \sinh \left(y /\left(1-(2 c)^{-1}\right)\right) \leq\left(\frac{c}{2}\right) e^{y /\left(1-(2 c)^{-1}\right)}$. Let $c=y / 2>1$, then

$$
\begin{aligned}
u(x, y) & \leq \frac{y}{4} \cdot e^{y /\left(1-y^{-1}\right)}=\frac{y}{4} e^{y\left(1+\frac{1}{y}+\frac{1}{y^{2}}+\ldots\right)} \\
& \leq(e+O(1 / y)) \frac{y}{4} e^{y} \quad \text { as } y \rightarrow \infty .
\end{aligned}
$$

EXAMPLE 3.4. In the case of catenoid, $u=\sqrt{(\cosh y)^{2}-x^{2}}$ and the defined domain $\Omega=\{-\cosh y<x<\cosh y \mid y>0\}$. Since $\Omega \subset\{-\sinh (y+1)<x<$ $\sinh (y+1) \mid y>-1\}$ and $\left.u\right|_{\partial \Omega} \leq c \sinh (y+1)$, where $c>1$. By Example 3.3, we have $u(x, y)=O\left((y+1) \cdot e^{y+1}\right)=O\left(y e^{y}\right)$.

REMARK 3.5. In Example 3.4, the actual growth behavior is $\cosh y=O\left(e^{y}\right)$, and our estimate $O\left(y e^{y}\right)$ are not optimal. In fact, the slower the growth of $u$ on $\partial \Omega$, the poorer the estimates of $u$ in $\Omega$.

ACKNOWLEDGMENTS. The author would like to thank the referee for many helpful comments and suggestions. 


\section{REFERENCES}

1. P. Aviles, Phragmén-Lindelöf and nonexistence theorems for nonlinear elliptic equations, Manuscripta Math. 43 (1983), 107-129.

2. A. Friedman, On two theorems of Phragmén-Lindelöf for linear elliptic and parabolic differential equations of the second order, Pacific J. Math. 7 (1957), 1563-1575.

3. S. Granlund, A Phragmén-Lindelöf principle for subsolutions of quasi-linear equations, Manuscripta Math. 36 (1981), 355-365.

4. D. Gilbarg and N.S. Trundinger, Elliptic partial differential equations of second order, SpringerVerlag, New York, 1977.

5. D. Gilbarg, The Phragmén-Lindelöf theorem for elliptic partial differential equations, J. Rational Mech. Anal. 1 (1952), 411-417.

6. E. Hopf, Remarks on the preceding paper of D. Gilbarg, J. Rational Mech. Anal. 1 (1952), 418-424.

7. K. Miller, Extremal barriers on cones with Phragmén-Lindelöf theorems and other applications, Ann. Mat. Pura Appl. 90 (1971), 297-329.

8. J. C. C. Nitsche, On new results in the theory of minimal surfaces, Bull. Amer. Math. Soc. 71 (1965), 195-270.

9. —_, Vorlesungen über Minimalflächen, Springer-Verlag, Berlin-Heidelberg-New York, 1975.

10. A. A. Novruzov, On theorems of Phragmén-Lindelöf type for solutions of second-order linear and quasilinear elliptic equations with discontinuous coefficients, Soviet Math. Dokl. 26 (1982), 376-379.

11. R. Osserman, A survey of minimal surfaces, Van Nostrand-Reinhold, New York, 1969.

12. M. H. Protter and H. F. Weinberger, Maximum principles in differential equations, PrenticeHall, Englewood Cliffs, N. J., 1967.

13. J. Serrin, On the Phragmén-Lindelöf principle for elliptic differential equations, J. Rational Mech. Anal. 3 (1954), 395-413.

Institute of Mathematics, ACademia Sinica, Nankang, Taipei 11529, Taiwan, REPUBLIC OF CHINA 